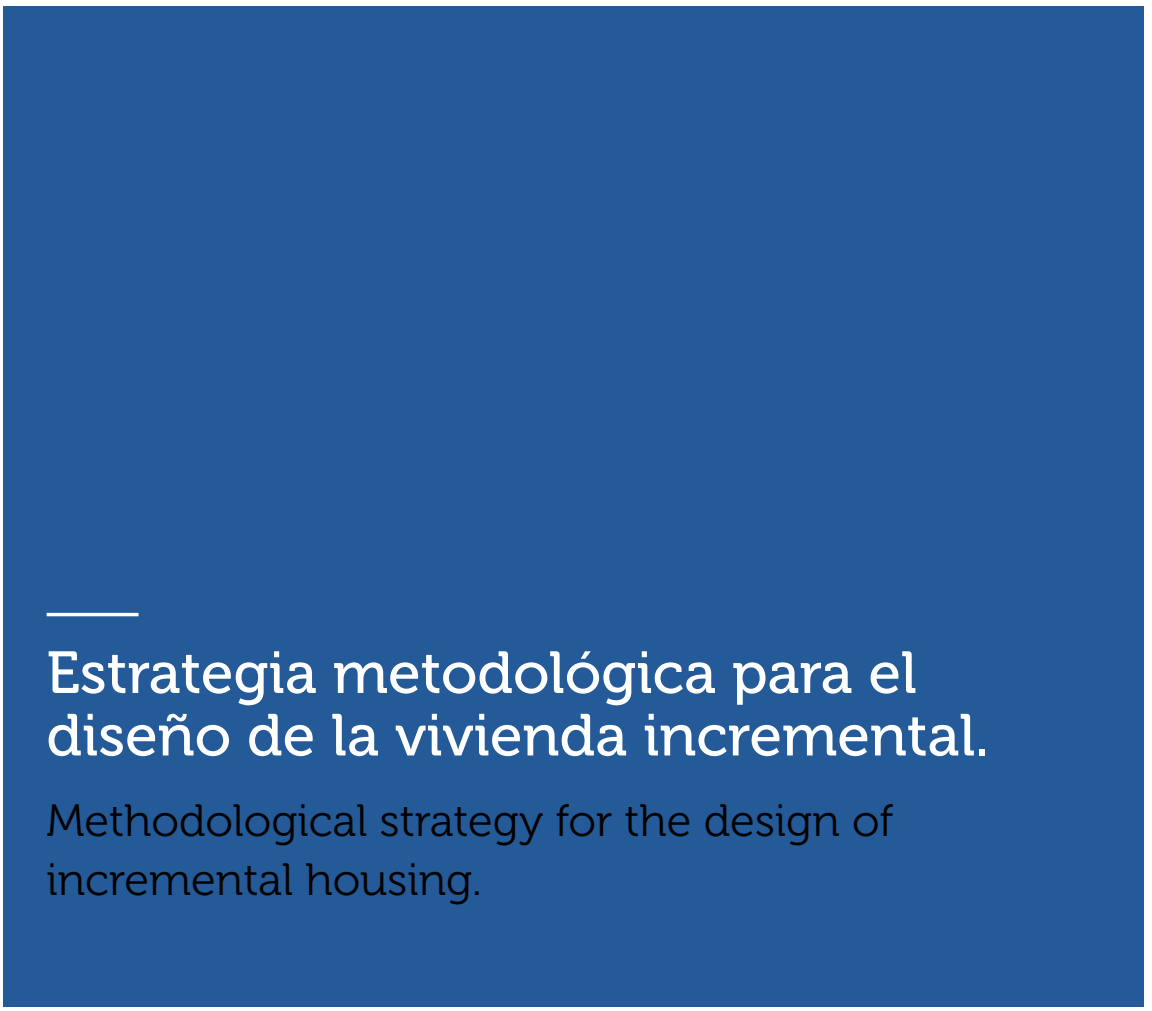

- Palabras clave/ Vivienda incremental, metodología de proyecto, Open Building

$\Delta$ Keywords/ Incremental housing, project method, Open Building

A Recepción/ 15 octubre 2015

A Aceptación/ 3 diciembre 2015

\title{
Bárbara Rangel
}

Arquitecta, Universidade de Oporto.

Magíster en Construcción de Edificios, Facultad de Ingeniería, Universidad de Oporto. Doctora en Ingeniería Civil, Faculdad de Ingeniería, Universidad do Oporto.

Académica, Departamento de Ingeniería Civil, Facultad de Ingeniería de la Universidad de Oporto.

brangelafe.up.pt

RESUMEN/ La actual crisis está en hacer que el concepto de 'casa' vuelva a su origen más elemental. La casa ya no es un artículo 'desechable'; tiene que desarrollarse de acuerdo con las necesidades de la familia, aumentando y disminuyendo de tamaño según sus necesidades. A esto se le ha llamado vivienda incremental y, en ella, el proyecto define las normas de evolución y el habitante va construyendo de acuerdo con las reglas de crecimiento definidas por el proyecto. En este artículo se organiza una estrategia de apoyo a la concepción arquitectónica en el momento del primero trazo. Recorriendo los impulsores de la investigación de esta tipología, se apunta a una en particular: Diseño de Soportes de SAR y Open Building. Se explican los criterios de diseño y se establecen los principios del proyecto. A partir de las obras de Elemental en Chile se hace la validación de la eficacia de esta opción. ABSTRACT/ The current crisis lies in shifting the concept of "house" to its most elemental roots. The house is no longer a "disposable" item; it needs to develop according to the needs of the family, growing and shrinking in size based on its requirements. This is what has been called incremental housing, where the project defines the standards for evolution and the dweller builds according to the growth rules defined in the project. This paper arranges a strategy that supports the architectural conception as from the first line. In reviewing the research promoters for this typology, a particular one emerges: Design criteria are explained and the project's principles are established. As from the Elemental works in Chile, the efficiency of this option is validated.

\section{INTRODUCCIÓN.}

"Un soporte es cualquier edificio hecho para contener un número determinado de unidades de vivienda, que puedan ser individualmente adaptadas a las necesidades cambiantes y a los deseos de los usuarios en el transcurso del tiempo" (Habraken 2015) En las últimas décadas, la industria de la construcción de viviendas prefabricadas ha entendido que el mercado ya no desea casas listas para ser habitadas, iguales a tantas otras (Montes, Camps y Fúster Abril 2011). La casa debe funcionar como un conjunto articulado de sistemas, tal como un ordenador; cada pieza debe poder ser fácilmente sustituida. Esto se debe a que separando los sistemas se responde con mayor eficacia a las exigencias de desempeño, se agiliza el proceso constructivo y se facilita la manutención (Rush 1986).

Fue solo en los años 60, cuando la vivienda económica pasó a ser una preocupación social para los arquitectos, que se empezó a investigar nuevas tipologías de habitación incremental prefabricada (Till, Wigglesworth, Schneider, Sherwood 2002). A partir de estudios realizados con las municipalidades o las cooperativas de vivienda, se desarrolló el diseño de soportes; un método que proponía una visión integradora desde la escala urbana hasta la definición del proceso constructivo, publicado en 1971 en "Variations: The Systematic Design of Supports" (Habraken 1979). Esta metodología fue adoptada en 


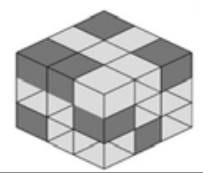

\begin{tabular}{|l|l|l|}
\hline SOPORTE & UNIDAD DE HABITACIÓN & UNIDAD SEPARABLE \\
\hline ESTRUTURA & CASA & EQUIPAMENTO ELETRICO \\
SUPER-ESTRUTRA & CONJUNTO DE UNIDADES & COCINA \\
INFRA-ESTRUTURAS & SEPARABLES & LAVABO \\
$\ldots$ & & ESCALERAS \\
& & VENTANAS \\
\hline
\end{tabular}

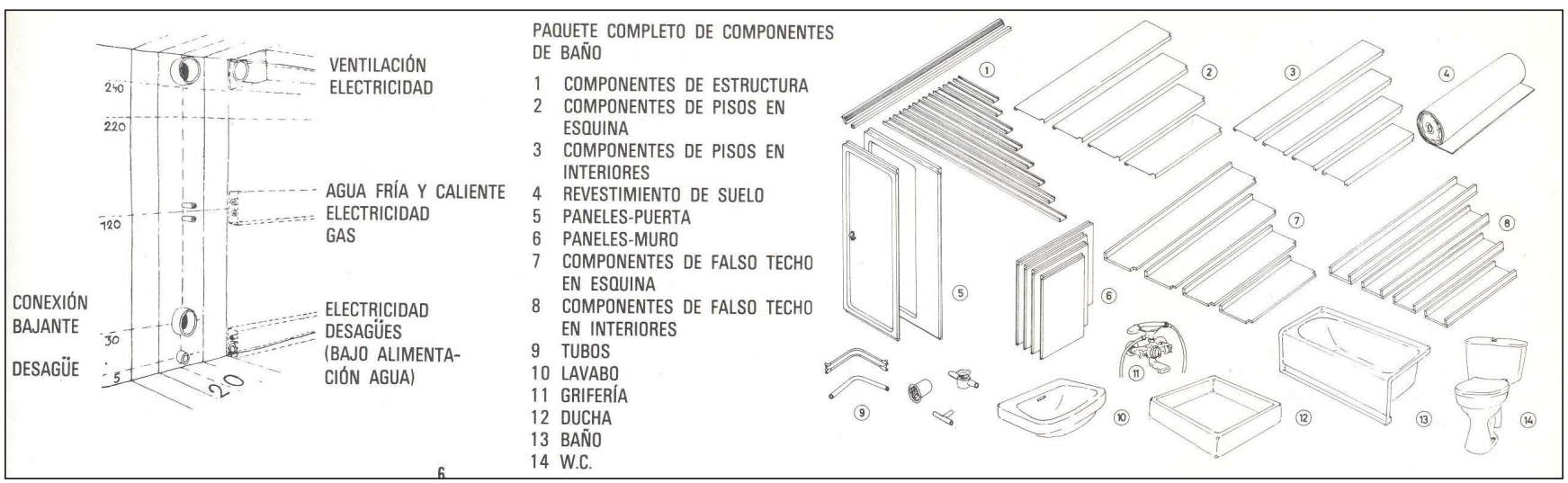

Imagen 1. Unidad Separable. Sistemas constructivos e infraestructuras (fuente: Kendall y Teicher 1999).

muchos proyectos de vivienda realizados en diferentes países (Habraken 1998), desde el Servicio Ambulatorio de Apoyo Local (SAAL) en los años 60 en Portugal (Duarte 2007), el Proyecto de Vivienda Experimental (PREVI) en la década del 70 en Lima (García-Huidobro, Torres y Tugass 2009), hasta el actual programa Chile Barrio (Gobierno de Chile 2003).

Al final de los años 90, la metodología fue seguida por el grupo Open Building, del equipo de trabajo W104 del Consejo Internacional para la Investigación y la Innovación en Edificación y Construcción (CIB 2006), con sede en el Instituto Tecnológico de Massachusetts (MIT) (Kendall y Teicher 1999). Allí pretendieron llevar el concepto de espacio abierto (open-space) más lejos y adoptarlo para los edificios residenciales, teniendo en cuenta que algunos espacios utilizados en la habitación pueden ser actualizados en determinado ciclo de tiempo. Esta investigación resultó en instrumentos de apoyo tanto para el diseño arquitectónico, como para las ingenierías, como es el caso del manual Residential Open Buildings (Kendall y Teicher 1999).

Es en Chile, por su tradición social, que se continúa incentivando a los alumnos a explorar esta tipología, tanto en proyectos como analizando, por ejemplo, las ciudades donde el diseño cívico fue el instrumento para el desarrollo de la ciudad (Arnstein 1969). Con el estudio de la ciudad de Talca, víctima del terremoto de 2010 en Chile, fue posible recuperar la ciudad intermedia y entender la importancia que tienen el sentido de barrio y de comunidad para la cohesión social (Inzulza 2014).

En este artículo se apunta a una estrategia de diseño de arquitectura que pueda auxiliar el proyecto de vivienda incremental, justo en el momento en que se hacen los primeros trazos después de asimilada la información obtenida del debate participativo con los ciudadanos. Después de interiorizados estos criterios y principios, será posible desarrollar el proyecto de una forma consciente y sustentada, considerando para ello las estrategias de optimización que los métodos computacionales permiten en la

\section{actualidad (Lyon y García 2013).}

\section{CRITERIOS DE DISEÑO.}

"Un soporte se diseña para prevenir la posibilidad de variar la planta de la vivienda a lo largo del tiempo, pero al mismo tiempo el soporte debe ser capaz de acomodar viviendas que cumplan con los estándares normalmente aceptados para viviendas en cada sociedad particular" (Habraken 1979). El diseño del soporte es un problema de optimización y de previsión de las variantes que la unidad separable podría tener. Esas variantes deben ser controladas para que el crecimiento de la casa no desvirtúe el conjunto ni implique un gran esfuerzo financiero para el habitante. Para obedecer a esta estrategia se debe seguir los siguientes criterios: 


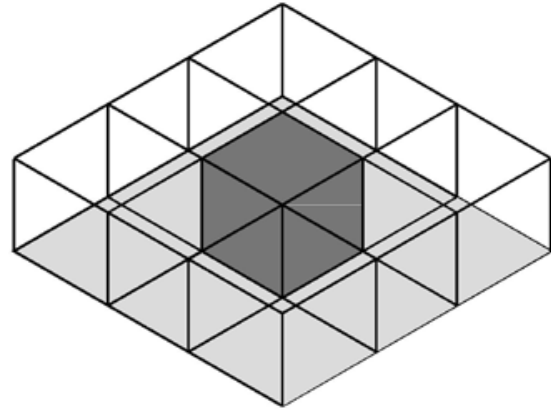

Imagen 2. Criterio de Diseño 2. Contenedor + contenido $=$ Soporte + unidades separables (fuente: Elaboración propia 2013).
1.1. CRITERIO DE DISEÑO 1: EDIFICIO = SOPORTE + UNIDAD DE HABITACIÓN + UNIDAD SEPARABLE.

El edificio se entiende como un soporte de varias unidades de habitación, compuestas por unidades separables adaptables para el uso pretendido. El soporte es el esqueleto del edificio; lo define el proyecto y lo dirige la comunidad. Las unidades de vivienda -la casa de cada familia- se componen por la articulación de las unidades separables. Dicha articulación es definida por el proyecto, y su montaje es de responsabilidad del habitante (tabla 1). Las unidades separables pueden ser consideradas como un conjunto de sistemas asociados a determinadas funciones -la cocina, el baño, etc.- y con la capacidad de ser construidas en varios soportes. Este concepto de unidad separable puede extenderse para los elementos constructivos como, por ejemplo, escaleras, paredes, etc. Cada una puede ser desarrollada separadamente y con contribuciones de diferentes proyectistas (imagen 1).

\subsection{CRITERIO DE DISEÑO 2: CONTENEDOR / CONTENIDO=} SOPORTE/ UNIDADES DE HABITACIÓN

Esta tipología se basa en el concepto de contenedor/contenido. El contenedor/ soporte es de propiedad de la comunidad de vecinos y el contenido/unidades de habitación de los habitantes (imagen 2) (Kendall y Teicher 1999). El soporte es un esqueleto capaz de contener varias soluciones de crecimiento, permitiendo varias formalizaciones para cada unidad de habitación, y, a su vez, cada unidad de habitación debe ser adaptable a diferentes soportes (Cuperus 2003).

El contenedor es la parte permanente del edificio, los servicios, redes de infraestructuras y áreas comunes. No se trata de un edificio inacabado, sino un edificio construido que será completado por diferentes unidades de habitación en el transcurso del tiempo. El contenido son los elementos que complementan el soporte y son fabricados por la industria.

\section{CRITÉRIO DE DISEÑO 3 NIVELES}

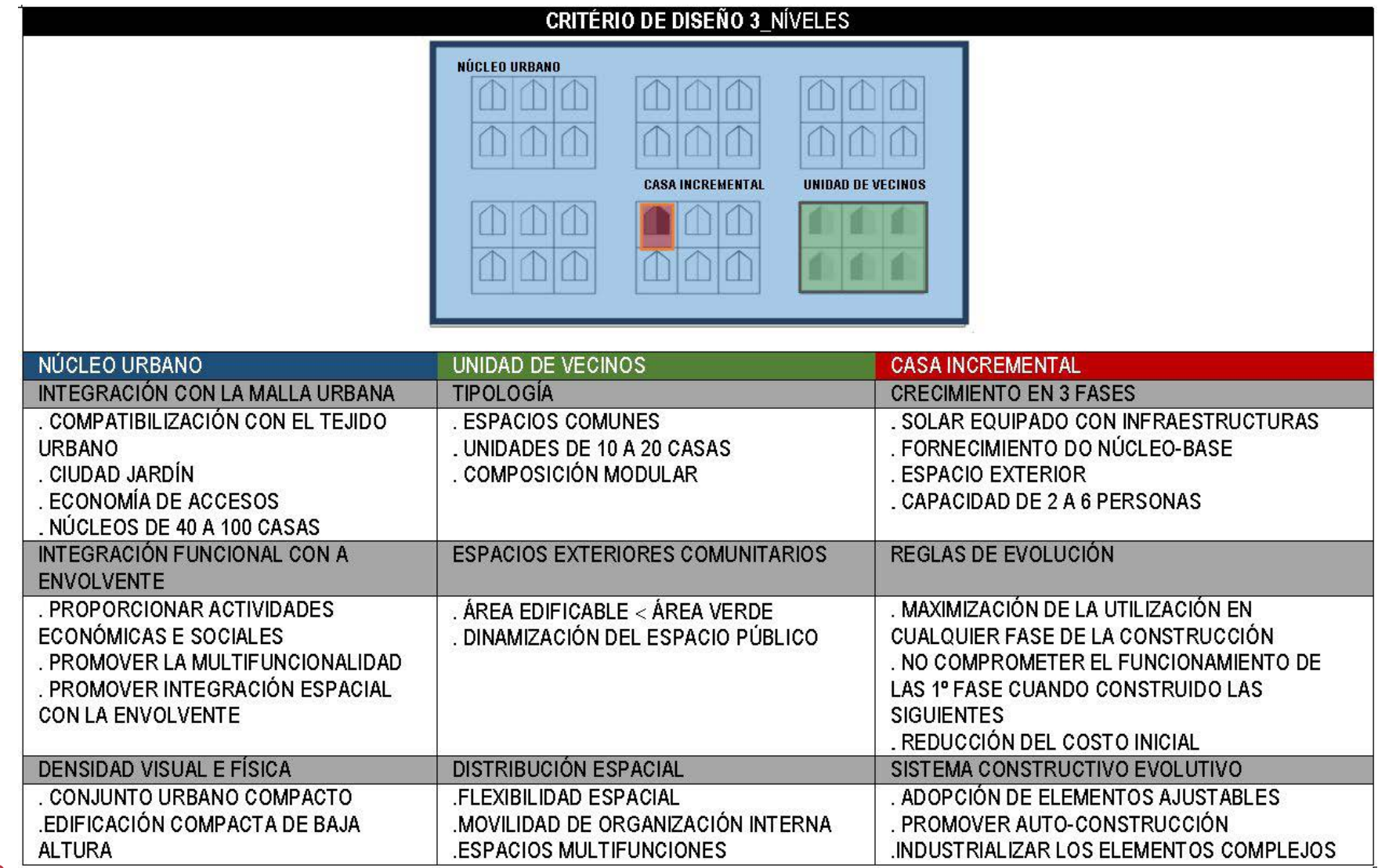


La construcción del soporte es más rápida y menos delicada, ya que la interacción de infraestructuras es menor, reduciéndose el tiempo de construcción. Se separa de una forma clara los problemas de índole tecnológica de las cuestiones logísticas y funcionales de la habitación.

\subsection{CRITERIO DE DISEÑO 3:}

NIVELES. Esta metodología de trabajo se basa en procesos de decisión que van descomponiendo el edificio en varios subsistemas flexibles, adaptados al uso futuro del habitante. Estos subsistemas se organizan en niveles definidos por las escalas del proyecto. El núcleo urbano establece la relación con la ciudad a la escala urbana. Mientras tanto, la unidad de vecinos articula las relaciones urbanas a la escala del barrio. La casa incremental define las relaciones funcionales, espaciales y constructivas en las distintas fases. En la tabla siguiente se sintetizan los principios a considerar para la definición de cada nivel (tabla 2).

La articulación de niveles apuesta a la eficacia de la relación contenedor/ contenido. Como el soporte podrá recibir distintas unidades, en el proyecto no se definen sistemas, sino el rendimiento que cada unidad separable debe tener. Cada unidad puede ser diseñada y concebida

separadamente con la contribución de distintas disciplinas (CIB W104 2006).

2. PRINCIPIOS DE PROYECTO. Estos

criterios se traducen en la organización de la información siguiendo un conjunto de

principios de proyecto.

\subsection{PRINCIPIOS DE PROYECTO 1: CATEGORÍAS ESPACIALES. Después}

de analizado el programa, se definen las dimensiones y la proximidad de los espacios, agrupándolos por categorías espaciales. Entre estas se encuentran los espacios de uso especial, para actividades específicas como dormir, estudiar o cocinar; espacios de uso general, que son espacios polivalentes para comer, estar y convivir; y los espacios de servicios, para actividades de corta duración, como higiene y circulación (tabla 3).

\subsection{PRINCIPIOS DE PROYECTO 2:} ZONAS DE INTERVENCIÓN.

el programa por categorías espaciales, se determinan los criterios de tolerancia de la concepción espacial, definiendo las zonas de intervención, que son las áreas necesarias para distribuir las funciones de acuerdo con la posición relativa a la fachada principal. Entre las zonas se definen los límites o las áreas de tolerancia espacial para articular esas funciones.
Se definen tres tipos de zonas de intervención: la zona o espacios de uso especial; la zona o espacios de servicios; y los sectores que establecen las relaciones funcionales entre las zonas de intervención

\section{(tabla 4).}

\subsection{PRINCIPIOS DE PROYECTO 3:} MALLA DE COMPOSICIÓN. Se puede definir la malla de composición tomando como referencia el patrón "tartan". una matriz de apoyo a la distribución del programa en el espacio disponible. Juntando las zonas de intervención y las categorías espaciales, se consigue obtener una mejor localización para cada espacio funcional (imagen 3).

PRINCIPIOS DE PROYECT01_CATEGORIAS ESPACIALES ESPACIOS DE USO ESPECIAL ESPACIOS DE DORMIR ESPACIOS DE ESTUDIAR ESPACIOS DE COCINAR

\section{ESPACIOS DE USO GENERAL ESPACIOS POLIVALENTES ESPACIOS PARA COMER} ESPACIOS ESTAR

\begin{tabular}{l|l} 
ESPACIOS PARA CONVIVIR \\
\hline
\end{tabular} ESPACIOS PARA ACTIVIDADES DE CORTA DURACIÓN ESPÁCIOS DE CUIDADOS DE HIGIENE

ESPÁCIOS DE CIRCULACIÓN

Tabla 3. Principios de Proyecto 1 Categorías Espaciales (fuente: Elaboración propia 2013).

PRINCIPIOS DE PROYECTO2 ZONAS DE INTERVENCIÓN

\begin{tabular}{|c|c|c|c|}
\hline ZONA a & NECESSÁRIA PROXIMIDADE A LA FACHADA & ESPACIOS DE USO ESPECIAL & \\
\hline & & & \\
\hline \multirow[t]{2}{*}{ SECTORES } & AREAS DE PROXIMIDAD ENTRE ZONAS & ESPACIOS DE USO GENERAL & \multirow{2}{*}{$\begin{array}{l}\text { ESPACIOS POLIVALENTES } \\
\text { ESPACIOS PARA COMER } \\
\text { ESPACIOS ESTAR } \\
\text { ESPACIOS PARA CONVIVIR }\end{array}$} \\
\hline & & & \\
\hline ZONA $\beta$ & PUDE ESTAR EN ESPACIOS INTERIORES & ESPACIOS DE SERVICIOS & \multirow{2}{*}{$\begin{array}{l}\text { ESPACIOS PARA ACTIVIDADES } \\
\text { DE CORTA DURACIÓN } \\
\text { ESPÁCIOS DE CUIDADOS DE } \\
\text { HIGIENE } \\
\text { ESPÁCIOS DE CIRCULACIÓN }\end{array}$} \\
\hline & & & \\
\hline
\end{tabular}

Tabla 4. Principios de Proyecto 2. Zonas de intervención (fuente: Elaboración propia 2013). 


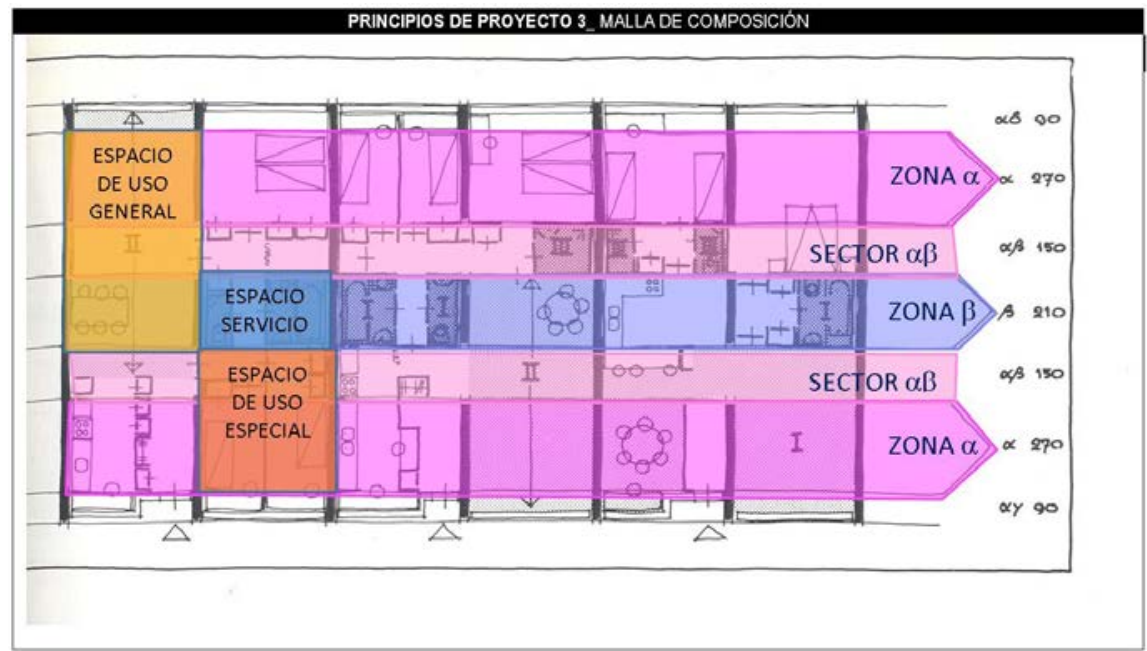

Imagen 3. Principios de Proyecto 3. Malla de composición, esquema ilustrativo de la distribución de las zonas de intervención (fuente: Elaboración propia 2013, en base a imagen de Habraken 1979).

\subsection{PRINCIPIO DE PROYECTO 4: DEFINICIÓN CONSTRUCTIVA DE \\ LAS DISTINTAS FASES. El principa}

objetivo de esta metodología es organizar el proyecto según la secuencia de las tres fases del proceso constructivo, de forma de garantizar la independencia de cada subsistema dentro del sistema principal sin interferir en el funcionamiento de los restantes. Las fases propuestas son las siguientes:

1" FASE - CONSTRUCCIÓN DEL SOPORTE/ CONTENEDOR:

20 FASE - MONTAJE DEL RELLENO/

CONTENIDO;
3 FASE - EVOLUCIÓN DE LAS

DIFERENTES UNIDADES SEPARABLES.

El proyecto requiere un conjunto de reglas para optimizar la interfase entre las diferentes disciplinas, las que se traducen en un conjunto de metas de proyecto para la definición constructiva (tabla 5):

\section{LA VERIFICACIÓN. PROYECTO ELEMENTAL.}

"The field of action is the city: the development of housing, public space, infrastructure and transportation projects that can perform as an effective and efficient upgrade in the quality of life of the poor. ELEMENTAL operates in contexts of scarce resources, using the city as a source of equality, and moreover, as a shortcut to correct inequalities" '(Aravena 2012)

La fuerte tendencia sísmica de Chile, junto a las dificultades económicas y sociales que vivió a finales del siglo XX, asociadas a una tradición entre la población más carente de auto-construcción, han hecho surgir en dicho país una apuesta por la tipología incremental, impulsada desde el gobierno y por arquitectos (Fernandez-Galiano 2008; Muñoz 2007). El programa puesto en marcha por el gobierno para albergar a la población sin hogar, que vive en situaciones precarias, ha hecho surgir en los últimos años uno de los proyectos más importantes del panorama arquitectónico internacional. Se trata de ELEMENTAL, conducido por el reciente Premio Pritzker 2015, Alejandro Aravena

A fines de los años 80, la profunda descripción de la vivienda en Chile realizada por la Facultad de Arquitectura de la Universidad Católica de Santiago de Chile, y orientada por Joan MacDonald, permitió establecer los aspectos conceptuales a tener en cuenta para el modelo progresivo que se defendía (MacDonald 1987). Para construir un gran número de viviendas con un mínimo de recursos, se concluyó que las casas debían ser capaces de crecer o ampliarse (Palmer y Vergara 1990). Entonces, con la misma inversión se daba cabida a más familias. Se trató de una decisión política importante, que cambió radicalmente la situación de la vivienda económica en Chile (Division Técnica de Estudio y Fomento Habitacional 1992):

№ DE FAMILIAS = RECURSOS TOTALES COSTO DE UNA CASA ACEPTABLE 


\begin{tabular}{|c|c|c|}
\hline \multicolumn{3}{|c|}{ PRINCIPIOS DE PROYECT04_DEFINICION CONSTRUCTIVA } \\
\hline $\begin{array}{l}\text { OBJETIVOS } \\
\text { METODOLÓGICOS }\end{array}$ & \multicolumn{2}{|c|}{$\begin{array}{l}\text { - OPTIMIZAR LOS ELEMENTOS CONSTRUCTIVOS EN UNA GESTIÓN GLOBAL DE LOS DIFERENTES SUBSISTEMAS; } \\
\text { - REDUCIR EL TIEMPO DE CONSTRUCCIÓN; } \\
\text { - SISTEMATIZAR EL PROCESO CONSTRUCTIVO SIMPLIFICANDO LA PRODUCCIÓN; } \\
\text { - MINIMIZAR LAS TAREAS EN LA EVOLUCIÓN DE LA CONSTRUCCIÓN; } \\
\text { - PROMOVER LA INNOVACIÓN Y EL DESARROLLO INDUSTRIAL DE SUBSISTEMAS CONSTRUCTIVOS; } \\
\text { - SACAR PARTIDO DE LAS TECNOLOGIAS Y CONOCIMIENTOS LOCALES }\end{array}$} \\
\hline & CONTENEDOR & CONTENIDO \\
\hline $\begin{array}{l}\text { OBJETIVOS DE } \\
\text { PROYECTO }\end{array}$ & $\begin{array}{l}\text { - LIBERTAR EL ESPACIO DE INFRA-ESTRUCTURAS } \\
\text { - PREVENIR UTILIZACIONES POLIVALENTES } \\
\text { - CONCEPCIÓN MODULAR }\end{array}$ & $\begin{array}{l}\text { - EQUIPAMIENTOS TIPO PLUG-AND-PLAY } \\
\text { - ADOPTAR EQUIPAMIENTOS DE POCA MANUTENCIÓN } \\
\text { - UTILIZAR PAVIMENTOS O TECHOS FALSOS PARA EL PASO } \\
\text { DE INFRAESTRUCTURAS }\end{array}$ \\
\hline $\begin{array}{l}\text { REGLAS DE } \\
\text { COMPOSICIÓN }\end{array}$ & $\begin{array}{l}\text { - PERMITIR VARIOS LAYOUTS } \\
\text { - POSIBLE AUMENTAR LA ÁREA DE LA UNIDAD DE } \\
\text { HABITACIÓN DENTRO DEL SOPORTE }\end{array}$ & $\begin{array}{l}\text { - PERMITIR VARIAS FORMALIZACIONES FINALES } \\
\text { - LOS SUBSISTEMAS INDEPENDIENTES DEL SOPORTE. } \\
\text { - ORGANIZAR LOS ESPACIOS POR KITS-MODULARES, TIPO } \\
\text { COCINA, BAÑO, ETC }\end{array}$ \\
\hline PERFORMANCE & $\begin{array}{l}\text { - SEGURIDAD } \\
\text { - DURABILIDAD } \\
\text { - FLEXIBILIDAD }\end{array}$ & $\begin{array}{l}\text { - SISTEMAS MODULARES } \\
\text { - CONCENTRACIÓN DE LAS INFRAESTRUCTURAS EN } \\
\text { DUCTOS TÉCNICOS }\end{array}$ \\
\hline $\begin{array}{l}\text { ELEMENTOS } \\
\text { CONSTRUCTIVOS }\end{array}$ & $\begin{array}{l}\text { - ESTRUCTURA CON EL NEGATIVO DE LAS } \\
\text { INFRAESTRUCTURAS } \\
\text { - FACHADA EXTERIOR } \\
\text { - DUCTOS } \\
\text { - PAVIMENTOS TÉCNICOS }\end{array}$ & $\begin{array}{l}\text { - } \text { INFRAESTRUTURAS } \\
\text { - } \text { EQUIPAMENTOS } \\
\text { - PAREDES DIVISÓRIAS } \\
\text { - } \text { MOBILIÁRIO } \\
\text { - } \text { CABINAS SANITÁRIAS } \\
\text { VANOS INTERIORES DE FÁCIL COLOCACIÓN }\end{array}$ \\
\hline $\begin{array}{l}\text { RESPONSABLE DE } \\
\text { MANTENIMIENTO }\end{array}$ & ENTIDAD PROMOTORA & $\begin{array}{l}\text { - HABITANTE } \\
\text { - SI POSIBLE IMPLEMENTAR SISTEMAS DE GESTIÓN DE } \\
\text { UTILIZACIÓN DE LOS SUBSISTEMAS. }\end{array}$ \\
\hline
\end{tabular}

Tabla 5. Definición Constructiva (fuente: Elaboración propia 2013).

Aprovechando la fuerte tradición chilena de autoconstrucción y la posibilidad de conseguir un subsidio estatal del Gobierno de Chile para adquirir una "vivienda dinámica social sin deuda”, el equipo de Aravena desarrolla con la población de distintas ciudades un proyecto para la construcción de conjuntos de viviendas sociales, con el apoyo del "Fondo

Concursable Solidario" (Knuckey 2008). El proyecto se basa en un modelo de tipologías incrementales capaces de adaptarse a diferentes contextos, siguiendo tres criterios de diseño principales: baja densidad de altura, capacidad moderada y posibilidad de crecimiento por autoconstrucción.

Invirtiendo la fórmula anterior, se fija el valor de cada casa y el área de su base, para determinar para cuántas familias se puede proporcionar alojamiento con un presupuesto específico:

PRESUPUESTO DISPONIBLE =

№ DE FAMILIAS X $\$ 7.500$ (subsidio para cada casa) $\times 30$ M
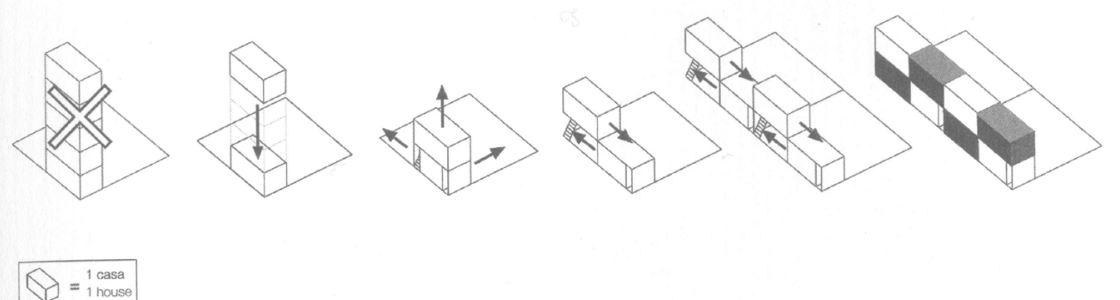

Imagen 4. ELEMENTAL. Concepto (fuente: Aravena 2012). 


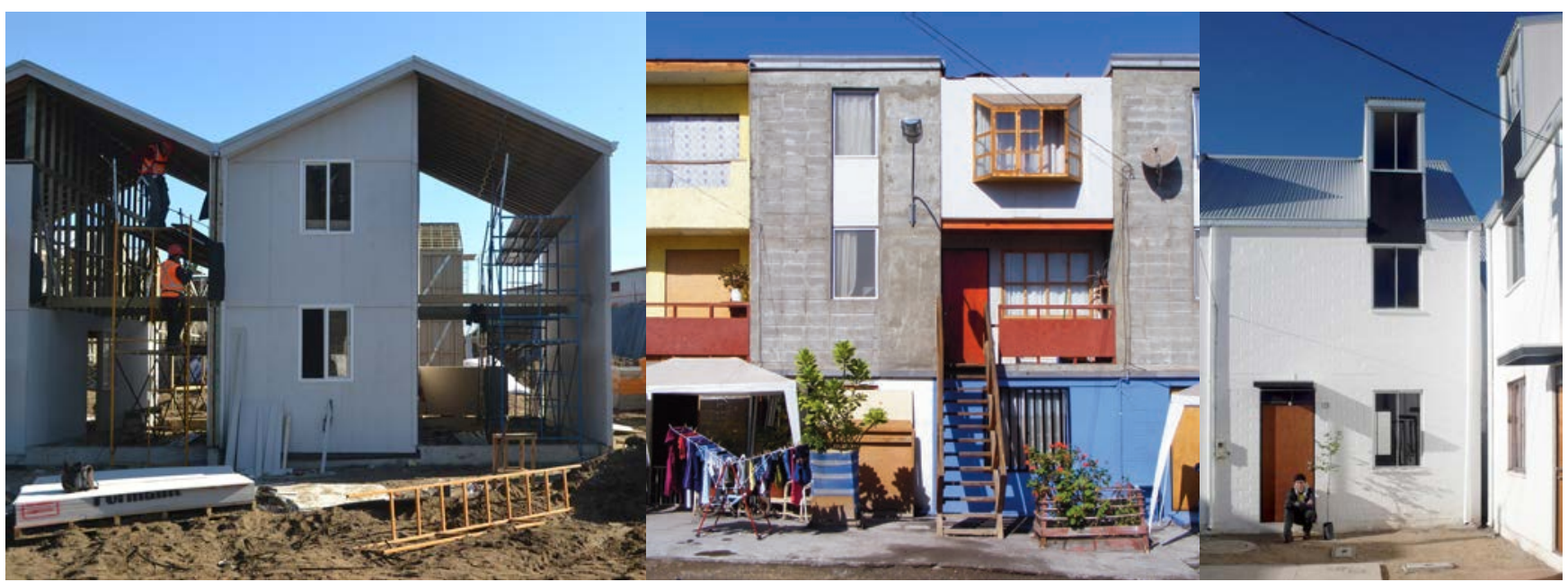

Imagen 5. ELEMENTAL. Casa ampliable, edificio paralelo y casa flexible (fuente: Aravena 2012).

Tomando como punto de partida una casa evolutiva desarrollada en tres módulos reducidos de $4,5 \mathrm{~m} \times 6,5 \mathrm{~m}$, se pueden definir núcleos urbanos adaptados a diferentes contextos, con unidades vecinales de 10 a 15 casas. La forma y área del terreno definirán la tipología de casa evolutiva a adoptar (imagen 4).

En la situación más elemental -un solar plano sin restricciones de área- se construye la primera mitad de la casa, la casa ampliable. Para un terreno reducido, se propone "un híbrido entre casa y edificio" (Aravena 2012) o edificio paralelo. En tres plantas, se sobreponen dos casas: la planta baja de la casa evoluciona en un piso, desarrollándose en la tipología dúplex. Para un terreno urbano se presenta una tipología expansible, construyéndose el casco exterior y posibilitándose en el interior la construcción de un piso más (imagen 5). Establecidos los principios de proyecto, se adapta el sistema constructivo de la casa evolutiva al mercado local. El núcleo base se compone de una estructura de hormigón o madera, cerrado por mampostería o paneles de madera de ejecución simple, para que se adapte fácilmente a las evoluciones futuras. Las reglas de las evoluciones se definen con cada comunidad durante el proyecto. El manual de instrucciones para mantener y hacer crecer la casa evolutiva se crea en conjunto con los ciudadanos (Aravena 2012). Este esfuerzo conjunto crea una gran complicidad entre las personas que participan en la expansión y mantenimiento de sus hogares y de los espacios

1. RESUMEN ESTRATÉGICO. Esta puede ser una herramienta para que las escuelas de arquitectura puedan hacer entender a los futuros profesionales los nuevos paradigmas de la vivienda (Vassigh y Chandler 2011). Después del análisis de estas metodologías, es importante aprovechar los conceptos que podrán ser adoptados en la elaboración del modelo operativo para la habitación incremental (Royal Institute of British Architects o RIBA 2010). Para apoyar la decisión del equipo de proyecto, se apunta un conjunto de estrategias metodológicas (tabla 6). 
2. CONCLUSIÓN. La vivienda incremental no es una corriente ni una forma de hacer arquitectura. Es un proceso metodológico de aproximación de los tres participantes principales en el proceso de construcción: proyectista, inversionista y habitante. Cuando se habla de proyecto participativo es importante organizar la metodología del proyecto y establecer responsabilidades. Después de entender la importancia del diseño participativo, es necesario estructurar una metodología de proyecto que pueda organizar el proceso constructivo de una forma consistente, siguiendo una estrategia de diseño organizada. En suma, una estrategia integrada, que busque una tipología de vivienda evolutiva y duradera, que armonice la industrialización de la construcción en la definición del contenedor y la apropiación individual en la construcción del contenido.

Esta tipología es tal vez la más fiel a la intervención participativa de los ciudadanos, pues les confía el crecimiento de cada casa para garantizar la integridad futura del conjunto. Ellos serán los responsables por su futuro y el de la comunidad en que viven. Para que el equipo responsable del proyecto se sienta confortable con esta complicidad, es importante establecer un camino conjunto definiendo las tareas de diseño, para que el proceso de construcción se armonice con el proceso evolutivo de cada casa, cada unidad de vecinos y cada núcleo urbano. Así, se podrá lograr un crecimiento de la ciudad realmente participativo.

\section{RESUMEN ESTRATÉGICO}

ESTRATÉGIA ECONOMICA

- Reducción de los costos inICIALLES DEL PROYECTO;

- otimizar LAS INVERSIONES FUTURAS;

- más RaPIDEZ EN La CONSTRUCCIÓn DEL SOPORTE;

- REDUCCIÓN DE LOS COSTOS DE COORDINACIÓN.

- Ventajas ECONÓmICAS a LARGo PLAZO;

- el EDIFICIO EStá pREPARAdo para ser técnicamente actualizado;

- REDUCCION DEL COSTO DE LA CONSTRUCCION EN LA TOTALIDAD;

- La pROPIEDAD DE la haBitación ES DNIDIDa. EL CONTENEDOR, DE MaYOR INVERSIÓN ES DEL INVERSIONISTA Y EL CONTENIDO DEL PROPIETARIO CONSTRUCCIÓN

- la evolución de la habitación es facilitada porque no depende de mano de la obra ESPECIALIZADA

- Se consigue así reducir el tiempo de obra porque la fase de terminaciones quedará

DRÁSTICAMENTE REDUCIDA;

- INDUSTRIALIZAR RACIONALMENTE LA HABITACIÓN;

- PERIODO DE OBRA DE LA SUPERESTRUTURA Y INFRAESTURCTURA MÁS REDUCIDO

\section{CRITEPIPS DE DISEÑO}

- conTENEDOR COMO ESQUEIETO/CONTENIDO COMD SUBSISTEMAS.

- aRTICULACIÓN DE LA INFORMACIÓN TRIDIMENSIONALMENTE

- DISENÕ PARTICIPATIVO

- aLta densidad con ba ja altura permitiendo a las famlias a permanecer cer

TRABAJAN.

- CUANDO EL NÚMERO DE VIVIENDAS SUPERA LOS 120, SE DIVIDE EN VARIOS NÚCLEOS;

- CUANDO EL TERRENO Y LA TOPOGRAFÍA ACCIDENTADA, Y PREFERIBLE UNA BANDA VOLUMETRÍA COMPACTA

UNIDAD VECINAL

- PREDECIR LA DUPLICACIÓN DE LASUPERFICIE BRUTAPOR VIVIENDAEN EL DISEÑO DEL ESPACIO URBANO

- cONCEBIR EL CONJUNTO CON LA PREVISIÓN QUE PUEDE CRECERENDIFERENTES MOMENTOS,

- eN PARCELAS PLANAS SE DEBE CENTRALIZAR LOS ESPACIOS PÚBLICOS

- INTERFACE ENTRE LOS COMPONENTES DEL CONTENIDO

- CONCENTRACIÓN DE NÚCLEO HÚMEDO CUARTO DE BAÑo Y COCINA

- sacar partido de los subsistemas industrializados para la definición del contenido

- paRa la segunda fase SE DEJAN LOS doRMITORIOS SUPLEMENTARES Y LA EXTENSIÓN DE LOS ESPACIOS COMUNES;

- para la segunda fase se debe optaR por sistemas locales de auto-Construcción.

- CREACIÓN DEL MANUAL DE INSTRUCCIONES.

Tabla 6. Resumen Estratégico (fuente: Elaboración propia 2013).

\section{REFERENCIAS}

Aravena, A., 2012. Elemental manua/ de vivenda incremental y diseño participativo. Ostfildern: Hatje Cantz. Arnstein, S.1969. "A Ladder of Citizen Participation."' Journal of the American Institute of Planner, Julio, 216-224

Gobierno de Chile. 2003. Programa Chile Barrio. Santiago de Chile: Ministerio de Vivienda y Urbanismo (MINVU) - Comisión Económica para América Latina (CEPAL).

Cuperus, Y., 2003. "Mass Customization in Housing an Open Building/ Lean Construction Study." Dense Living Urban Structures International Conference on Open Building, Hong Kong.

Division Técnica de Estudio y Fomento Habitacional, 1992. Vivenda Progressiva - Programa 1990. Santiago de Chile: Ministerio da Vivienda y Urbanismo.

Duarte, J., 2007. Personalizar a habitação em série uma gramática discursiva para as casas da Malagueira do Siza, Textos universitários de Ciências Sociais e Humanas. Lisboa: Fundação Calouste Gulkenkian Fundação para a Ciência e a Tecnologia

Fernandez-Galiano, L., 2008. "Paisaje después de la Borbuja." Arquitectura Viva, 122.

García-Huidobro, F., Torres, D. y Tugass, N., 2009. "PREVI Lima y la experiencia del tiempo." Revista beroamericana de Urbanismo.

Habraken J. 2015. John Habraken home page http://www habrakencom/ (13.1213).

Habraken, J., 1998. The Structure of The Ordinary: Form and Control in the Built Environment. MIT Press.

Habraken, J., 1979. El diseño de soportes. Barcelona. Gustavo Gili.

Inzulza, J. 2014، "La recuperación del diseño civico como reconstrucción de lo local en la ciudad intermedia: el

caso de Talca, Chile." Revista AUS 15, 4-8.

International Council for Research and Innovation in Building and ConstructionCIB W104. 2006. "Open

Building Implementation." http://www.open-building.org/ob/concepts.html
Kendall, S., y Teicher, J., 1999. Residential Open Buildings. London, GBR: Spon Press.

Knuckey, J., 2008. Elemental TV Commercial MCM Cine.

Lyon, A. y Garcia, R., 2013. "Forma a topológica: Nuevos métodos para antiguos problemas." AUS Arquitectura/Urbanismo/Sustentabilidad, 27-30

MacDonald, J. 1987. Vivienda progresiva. Santiago de Chile: Corporación de Promoción Universitaria.

Montes, J., Camps, I. y Füster, A., 2011. "Industralización en la vivenda social en Madrid." Informes de la construcción 63 (522), 5 -19.

Palmer, M., y Vergara, F., 1990. El lote de 9 × 18. Santiago de Chile: Edit. Universidad Católica.

Muñoz, C., 2007. "Vivienda Progresiva, un programa del sector público que se potenció en el hábitat rurał

chileno." Revista INVI, 22 (59),151-167. Recuperado de http://www.revistainvi.uchile.cl/index.php/INVI/article/ view/293/267.

Rangel, B. 2013 proposta metodológica para o desenvolvimento do projeto integrado de habitacão evolutiva em portugal. (Tesis doctoral, Departamento de Engenharia Civil, Porto).

Royal Institute of British Architects (RIBA). 2010. Architect's handbook of practice management. Vol. 8th ed Royal Institute of British Architects. London: RIBA Publications.

Rush, R., 1986. The Building Systems Integration Handbook. John Wiley \& Sons Inc.

Sherwood, R. 2002. Housing prototypes. Recuperado de: http://housingprototypes.org/

Till, J., Wigglesworth, S., y Schneider, T., 2002. Flexible Housing Project. Recuperado de: http://www.afew-

thoughts.co.uk/flexiblehousing/index.php (26.04.11)

Vassigh, S. y Chandler, J. 2011. Building Systems Integration for Enhanced Environmental Performance. J. Ross

Publishing. 